\title{
Definition System and Criteria Related to the Static Stability of Airplanes (Third Report) —Static Sideslip Stability, Static Roll Stability, and Static Yaw Stability-
}

\author{
By Osamu KoBAYASHI \\ Department of Aeronautics and Astronautics, Tokai University, Hiratsuka, Japan
}

(Received November 6th, 2001)

\begin{abstract}
To systematically study the static stability for airplanes, we proposed the system of definitions of six static stability concepts. The details of "static flight speed stability" and "static angle of attack stability" were discussed in the first report of this paper, and "static pitch stability" was studied in the second. In this third report, the static stability concepts of lateral and directional motions are discussed, and their stability criteria are obtained in terms of the aerodynamic characteristics of an airframe. They are "static sideslip stability," "static roll stability," and "static yaw stability," and their fundamental definitions are respectively " $\mathrm{d} Y / \mathrm{d} \beta<0$," " $\mathrm{d} R / \mathrm{d} \Phi<0$," and " $\mathrm{d} N / \mathrm{d} \Psi<0$," where $R$ denotes rolling moment. The general criteria are deduced from these definitions. Moreover, they give " $C_{Y \beta}<0$ " for static sideslip stability, " $C_{\ell \beta} / C_{Y \beta}>0$ " for static roll stability, and " $C_{n \beta}>0$ " for static yaw stability, respectively, as the criteria in normal and low subsonic flights. As a conclusion, a summary of this entire paper is given.
\end{abstract}

Key Words: Aircraft, Stability and Control, Static Stability

$$
\begin{aligned}
& \text { Nomenclature } \\
& b \text { : wing span } \\
& C_{D}, C_{L}, C_{m} \text { : coefficients of drag, lift, and pitching mo- } \\
& \text { ment } \\
& C_{L 1 \mathrm{~g}}: \quad \text { lift coefficient in a } 1 \mathrm{~g} \text { flight } \\
& C_{D u}, C_{L u}, C_{m u}: \quad \partial C_{D} / \partial(\Delta U / U), \partial C_{L} / \partial(\Delta U / U), \\
& \partial C_{m} / \partial(\Delta U / U) \\
& C_{D \delta_{e}}, C_{L \delta_{e}}, C_{m \delta_{e}}: \quad \partial C_{D} / \partial \delta_{e}, \partial C_{L} / \partial \delta_{e}, \partial C_{m} / \partial \delta_{e} \\
& C_{L \delta_{\mathrm{DLC}}}, C_{Y \delta_{\mathrm{DSFC}}}, C_{\ell \delta_{a}}, C_{n \delta_{r}}: \quad \partial C_{L} / \partial \delta_{\mathrm{DLC}}, \partial C_{Y} / \partial \delta_{\mathrm{DSFC}}, \\
& \partial C_{\ell} / \partial \delta_{a}, \partial C_{n} / \partial \delta_{r}
\end{aligned}
$$

DLC, DSFC : direct lift control and direct side force control

FC.0 : initial trimmed flight condition

FC.1 : steady flight condition after a flight variable of interest is changed from FC.0

FC.2 : nonequilibrium flight condition just after the change in primary control in FC. 1 is returned quickly to zero

$L$ : aerodynamic lift

$M, N$ : aerodynamic pitching moment and yawing moment
$R$ : aerodynamic rolling moment

$S: \quad$ wing area

$T: \quad$ thrust produced by propulsion system

$U:$ flight speed

$W: \quad$ weight of airplane

$Y:$ aerodynamic side force

$\alpha, \beta, \gamma:$ angle of attack, sideslip angle, and flight path angle

$\delta_{a}:$ aileron deflection (positive: right aileron down and left aileron up)

$\delta_{e}:$ elevator deflection (positive: elevator trailing edge down)

$\delta_{r}:$ rudder deflection (positive: rudder trailing edge left)

$\delta_{\text {DLC }}: \quad$ DLC deflection (positive: lift $L$ increases)

$\delta_{\mathrm{DSFC}}: \quad$ DSFC deflection (positive: side force $Y$ increases)

$\rho:$ air density

$\Theta, \Phi, \Psi$ : pitch, roll, and yaw attitude

$\Delta$ : small increment

Subscript 0, 1, 2 : FC.0, FC.1, and FC.2

\section{Introduction}

The definition of static stability appears not to be systematically discussed. For example, although " $C_{m \alpha}<0$," " $C_{\ell \beta}<0$," and " $C_{n \beta}>0$ " are merely the aerodynamic criteria corresponding to each static stability, they are used just like the definition of static stability. ${ }^{1-6)}$ Moreover, "flight path stability" is well known as the static stability concept on 
Table 1. Definition system of static stability.

\begin{tabular}{|c|c|c|c|c|}
\hline & \multirow{2}{*}{$\begin{array}{l}\text { Fundamental } \\
\text { definition }\end{array}$} & \multirow{2}{*}{$\begin{array}{c}\text { Derived form of } \\
\text { definition }\end{array}$} & \multicolumn{2}{|c|}{ Control equipment } \\
\hline & & & Primary control & Secondary control \\
\hline \multirow[t]{2}{*}{$\begin{array}{l}\text { Static flight speed } \\
\text { stability }\end{array}$} & \multirow{2}{*}{$\frac{\mathrm{d} D_{\text {net }}}{\mathrm{d} U}>0$} & $\frac{\mathrm{d} T}{\mathrm{~d} U}>0$ & Propulsion system & Elevator (Holding flight path angle) \\
\hline & & $\frac{\mathrm{d} \gamma}{\mathrm{d} U}<0$ & $\begin{array}{l}\text { Gravity force component or flight path } \\
\text { angle }\end{array}$ & - \\
\hline $\begin{array}{l}\text { Static angle of attack } \\
\text { stability }\end{array}$ & $\frac{\mathrm{d} L}{\mathrm{~d} \alpha}>0$ & $\frac{\mathrm{d} \delta_{\text {DLC }}}{\mathrm{d} \alpha}<0$ & DLC system & Elevator (Holding flight path angle) \\
\hline Static pitch stability & $\frac{\mathrm{d} M}{\mathrm{~d} \Theta}<0$ & $\frac{\mathrm{d} \delta_{e}}{\mathrm{~d} \Theta}<0$ & Elevator & - \\
\hline \multirow[t]{2}{*}{ Static sideslip stability } & \multirow{2}{*}{$\frac{\mathrm{d} Y}{\mathrm{~d} \beta}<0$} & $\frac{\mathrm{d} \delta_{\mathrm{DSFC}}}{\mathrm{d} \beta}>0$ & DSFC system & $\begin{array}{l}\text { Aileron/rudder (Holding roll attitude } \\
\text { and straight flight) }\end{array}$ \\
\hline & & $\frac{\mathrm{d} \Phi}{\mathrm{d} \beta}>0$ & $\begin{array}{l}\text { Gravity force component or roll atti- } \\
\text { tude }\end{array}$ & Rudder (Holding straight flight) \\
\hline Static roll stability & $\frac{\mathrm{d} R}{\mathrm{~d} \Phi}<0$ & $\frac{\mathrm{d} \delta_{a}}{\mathrm{~d} \Phi}<0$ & Aileron & Rudder (Holding straight flight) \\
\hline Static yaw stability & $\frac{\mathrm{d} N}{\mathrm{~d} \Psi}<0$ & $\frac{\mathrm{d} \delta_{r}}{\mathrm{~d} \Psi}<0$ & Rudder & Aileron (Holding straight flight) \\
\hline
\end{tabular}

one of the translational motions, ${ }^{7,8)}$ but two static stability concepts on heaving and sliding motions are not discussed systematically. And "tuck under",5) and "flight path divergence"10) are also not discussed on the basis of the definition system on static stability, but they are individually treated as special problems.

So the first report ${ }^{11)}$ of this paper proposed the definition system consisting of six static stability concepts. They are "static flight speed stability," "static angle of attack stability," "static pitch stability," "static sideslip stability," "static roll stability," and "static yaw stability." In the first report of this paper, "static flight speed stability" and "static angle of attack stability" were discussed in detail. In the second report, ${ }^{12)}$ "static pitch stability" was studied in regard to its derived forms of definition and its criteria in terms of the aerodynamic characteristics of an airplane. The definition system proposed in the first and second reports is shown in Table 1.

This third report discusses in detail the other three static stability concepts that are commonly called "lateral and directional static stability." They are "static sideslip stability," "static roll stability," and "static yaw stability." In conclusion, a summary of this entire paper is presented.

\section{Static Sideslip Stability}

In this chapter, the detail of "static sideslip stability" is discussed in the two instances using a direct side force control (DSFC) system or a gravity force component as primary control, and its stability criteria in terms of the aerodynamic characteristics of airframe are deduced.

\subsection{Using a DSFC system as primary control}

The procedures in flight tests for judging static sideslip stability are as follows. We suppose as a starting point that an airplane is flying in a trimmed straight and wing-level flight condition (initial flight condition "FC.O").

To give a small change to the sideslip angle in FC.0, a side force as an external force is applied to the airplane by using its DSFC system (a main control called "primary control"), maintaining steady straight flight and roll attitude by using its aileron and rudder (a subsidiary control called "secondary control"). As a result, the new steady flight condition in which the sideslip angle $\beta_{1}$ is " $\beta_{0}+\Delta \beta$ " is established (a flight condition called "FC.I"). The flight speed and flight path angle in FC.1 are assumed to be equal to those in FC.0, (i.e., $U_{1}=U_{0}$ and $\gamma_{1}=\gamma_{0}$ ).

The additive DSFC deflection $\Delta \delta_{\mathrm{DSFC}}$ is then quickly returned to zero (an instantaneous flight condition called "FC.2"). At this FC.2, if the nonequilibrium side force $\Delta Y$ results to act as the restoring force to the increment in sideslip angle $\Delta \beta$, the static sideslip stability is stable, and vice versa. Therefore the condition for static sideslip stability to be stable is given by

$$
\mathrm{d} Y / \mathrm{d} \beta<0
$$

This is the fundamental definition of "static sideslip stability."

The side force applied by the DSFC system in FC.1 balances against $\Delta Y$ mentioned above (i.e., $\left[\rho U_{2}^{2} / 2\right] S b$. $C_{Y \delta_{\mathrm{DSFC}}} \Delta \delta_{\mathrm{DSFC}}=-\Delta Y$ ). This means that the static stability can be judged by checking the sense of the primary control in FC.1. Consequently, since this paper defines $C_{Y \delta_{\mathrm{DSFC}}}$ to be positive, the derived form of definition of static sideslip stability is given by

$$
\mathrm{d} \delta_{\mathrm{DSFC}} / \mathrm{d} \beta>0
$$

In the following discussion, it is assumed for simplicity that the DSFC system is an aerodynamic control sur- 
face and that the change in DSFC deflection $\Delta \delta_{\mathrm{DSFC}}$ gives only the change in aerodynamic side force $\Delta Y_{\mathrm{DSFC}}$, that is, " $\Delta \delta_{\mathrm{DSFC}}=(1 / 2) \rho U^{2} S C_{Y \delta_{\mathrm{DSFC}}} \Delta \delta_{\mathrm{DSFC}}$." And as a general rule in this paper, the aerodynamic coefficients and derivatives without subscripts 0, 1, and 2 show the values in FC.0.

\subsubsection{Equilibrium equation and restoring force}

The equilibrium equations on side force $Y$, rolling moment $R$, and yawing moment $N$ in FC. 0 and FC. 1 are respectively described as follows.

"In FC.0":

Side force: $\quad Y_{0}=-W \cos \gamma_{0} \sin \Phi_{0}$,

or $C_{Y}=-C_{L 1_{q}} \cos \gamma_{0} \sin \Phi_{0}$

Rolling moment: $\quad R_{0}=0, \quad$ or $\quad C_{\ell}=0$

Yawing moment: $N_{0}=0, \quad$ or $\quad C_{n}=0$

"In FC.1":

Side force: $\quad Y_{1}=-W \cos \gamma_{0} \sin \Phi_{0}$,

or

$$
\begin{aligned}
C_{Y 1} & =C_{Y}+C_{Y \beta} \Delta \beta+C_{Y \delta_{a}} \Delta \delta_{a} \\
& +C_{Y \delta_{r}} \Delta \delta_{r}+\Delta C_{Y_{\mathrm{DSFC}}} \\
& =-C_{L 1_{g}} \cos \gamma_{0} \sin \Phi_{0},
\end{aligned}
$$$$
\text { or } \quad C_{Y \beta} \Delta \beta+C_{Y \delta_{a}} \Delta \delta_{a}+C_{Y \delta_{r}} \Delta \delta_{r}+\Delta C_{Y_{\mathrm{DSFC}}}=0
$$

Rolling moment: $\quad R_{1}=0$,

$$
\begin{aligned}
& \text { or } C_{\ell 1}=C_{\ell}+C_{\ell \beta} \Delta \beta+C_{\ell \delta_{a}} \Delta \delta_{a}+C_{\ell \delta_{r}} \Delta \delta_{r}=0, \\
& \text { or } \quad C_{\ell \beta} \Delta \beta+C_{\ell \delta_{a}} \Delta \delta_{a}+C_{\ell \delta_{r}} \Delta \delta_{r}=0
\end{aligned}
$$

Yawing moment: $\quad N_{1}=0$,

$$
\begin{aligned}
& \text { or } C_{n 1}=C_{n}+C_{n \beta} \Delta \beta+C_{n \delta_{a}} \Delta \delta_{a}+C_{n \delta_{r}} \Delta \delta_{r}=0, \\
& \text { or } C_{n \beta} \Delta \beta+C_{n \delta_{a}} \Delta \delta_{a}+C_{n \delta_{r}} \Delta \delta_{r}=0
\end{aligned}
$$

where

$$
\begin{aligned}
& \Delta C_{Y_{\mathrm{DSFC}}}=\Delta Y_{\mathrm{DSFC}} /\left(\rho U_{0}^{2} S / 2\right)=C_{Y \delta_{\mathrm{DSFC}}} \Delta \delta_{\mathrm{DSFC}} \\
& C_{L 1_{g}}=W /\left(\rho U_{0}{ }^{2} S / 2\right)
\end{aligned}
$$

The nonequilibrium side force $\Delta Y$ at FC.2, when the additive DSFC $\Delta \delta_{\mathrm{DSFC}}$ in FC. 1 is returned to zero, can be obtained by subtracting the side force component $\Delta C_{Y_{\mathrm{DSFC}}}$ produced by the DSFC system from the equilibrium condition " $C_{Y 1}+C_{L 1_{q}} \cos \gamma_{0} \sin \Phi_{0}=0$ " in FC.1 and is shown by

$$
\begin{aligned}
\Delta Y & =\rho U_{0}^{2} S\left(C_{Y 1}+C_{L 1_{g}} \cos \gamma_{0} \sin \Phi_{0}-\Delta C_{Y_{\mathrm{DSFC}}}\right) / 2 \\
& =\rho U_{0}^{2} S\left(C_{Y \beta} \Delta \beta+C_{Y \delta_{a}} \Delta \delta_{a}+C_{Y \delta_{r}} \Delta \delta_{r}\right) / 2
\end{aligned}
$$

By obtaining the change in side force $\Delta Y_{\mathrm{DSFC}}$ produced by the DSFC deflection from Eq. (4a) and (5a), we can reasonably show that this $\Delta Y_{\mathrm{DSFC}}$ balances against $\Delta Y$ (i.e., $\left.\Delta Y_{\mathrm{DSFC}}=-\Delta Y\right)$. Therefore because $C_{\ell \delta_{a}}<0$, the definitions Eq. (1) and Eq. (2) are equivalent.

\subsubsection{Criteria for static sideslip stability}

The total differential $\mathrm{d} z$ in the vicinity of point $(x, y, \cdots)$ of " $z=f(x, y, \cdots)$ " has the following relation.

$$
\mathrm{d} z=(\partial z / \partial x) \mathrm{d} x+(\partial z / \partial y) \mathrm{d} y+\cdots
$$

So because Eq. (6) shows that $\Delta Y$ is a function of $\Delta \beta, \Delta \delta_{a}$, and $\Delta \delta_{r}$, the definition Eq. (1) may be written

$$
\frac{\mathrm{d} Y}{\mathrm{~d} \beta}=\frac{\partial Y}{\partial \beta}+\frac{\partial Y}{\partial \delta_{a}} \cdot \frac{\mathrm{d} \delta_{a}}{\mathrm{~d} \beta}+\frac{\partial Y}{\partial \delta_{r}} \cdot \frac{\mathrm{d} \delta_{r}}{\mathrm{~d} \beta}<0
$$

The partial differentials in Eq. (8) are given by differentiating Eq. (6) as follows:

$$
\begin{aligned}
& \partial Y / \partial \beta=\rho U_{0}^{2} S \cdot C_{Y \beta} / 2 \\
& \partial Y / \partial \delta_{a}=\rho U_{0}^{2} S \cdot C_{Y \delta_{a}} / 2 \\
& \partial Y / \partial \delta_{r}=\rho U_{0}{ }^{2} S \cdot C_{Y \delta_{r}} / 2
\end{aligned}
$$

The total differentials $\mathrm{d} \delta_{a} / \mathrm{d} \beta$ and $\mathrm{d} \delta_{r} / \mathrm{d} \beta$ in Eq. (8) are deduced by solving the simultaneous equations of Eq. (4a) $\sim(4 \mathrm{c})$ to obtain the ratios of $\Delta \beta, \Delta \delta_{a}$, and $\Delta \delta_{r}$ to $\Delta C_{Y_{\mathrm{DSFC}}}$ as follows:

$$
\begin{aligned}
& \Delta \beta / \Delta C_{Y_{\mathrm{DSFC}}}=\left(C_{n \delta_{a}} C_{\ell \delta_{r}}-C_{\ell \delta_{a}} C_{n \delta_{r}}\right) / A \\
& \Delta \delta_{a} / \Delta C_{Y_{\mathrm{DSFC}}}=\left(C_{\ell \beta} C_{n \delta_{r}}-C_{n \beta} C_{\ell \delta_{r}}\right) / A \\
& \Delta \delta_{r} / \Delta C_{Y_{\mathrm{DSFC}}}=\left(C_{n \beta} C_{\ell \delta_{a}}-C_{\ell \beta} C_{n \delta_{a}}\right) / A
\end{aligned}
$$

where

$$
\begin{aligned}
A & =C_{Y \beta}\left(C_{\ell \delta_{a}} C_{n \delta_{r}}-C_{n \delta_{a}} C_{\ell \delta_{r}}\right)+C_{\ell \beta}\left(C_{n \delta_{a}} C_{Y \delta_{r}}-C_{Y \delta_{a}} C_{n \delta_{r}}\right) \\
& +C_{n \beta}\left(C_{Y \delta_{a}} C_{\ell \delta_{r}}-C_{\ell \delta_{a}} C_{Y \delta_{r}}\right)
\end{aligned}
$$

Thus $\mathrm{d} \delta_{a} / \mathrm{d} \beta$ and $\mathrm{d} \delta_{r} / \mathrm{d} \beta$ are obtained as follows:

$\frac{\mathrm{d} \delta_{a}}{\mathrm{~d} \beta}=\left(\frac{\Delta \delta_{a}}{\Delta C_{Y_{\mathrm{DSFC}}}}\right) /\left(\frac{\Delta \beta}{\Delta C_{Y_{\mathrm{DSFC}}}}\right)=\frac{C_{\ell \beta} C_{n \delta_{r}}-C_{n \beta} C_{\ell \delta_{r}}}{C_{n \delta_{a}} C_{\ell \delta_{r}}-C_{\ell \delta_{a}} C_{n \delta_{r}}}$

$\frac{\mathrm{d} \delta_{r}}{\mathrm{~d} \beta}=\left(\frac{\Delta \delta_{r}}{\Delta C_{Y_{\mathrm{DSFC}}}}\right) /\left(\frac{\Delta \beta}{\Delta C_{Y_{\mathrm{DSFC}}}}\right)=\frac{C_{n \beta} C_{\ell \delta_{a}}-C_{\ell \beta} C_{n \delta_{a}}}{C_{n \delta_{a}} C_{\ell \delta_{r}}-C_{\ell \delta_{a}} C_{n \delta_{r}}}$

When Eqs. (9a) (9c), (14a), and (14b) are applied to the definition Eq. (8), the stability criterion in terms of the aerodynamic characteristics of an airplane is given by

$C_{Y \beta}+\frac{C_{Y \delta_{a}}\left(C_{\ell \beta} C_{n \delta_{r}}-C_{n \beta} C_{\ell \delta_{r}}\right)+C_{Y \delta_{r}}\left(C_{n \beta} C_{\ell \delta_{a}}-C_{\ell \beta} C_{n \delta_{a}}\right)}{C_{n \delta_{a}} C_{\ell \delta_{r}}-C_{\ell \delta_{a}} C_{n \delta_{r}}}$

$<0$

Since the derivatives $C_{Y \delta_{a}}, C_{n \delta_{a}}, C_{Y \delta_{r}}$, and $C_{\ell \delta_{r}}$ are small and may be ignored for conventional airplanes, Eq. (15) is simplified as follows:

$$
C_{Y \beta}<0
$$

This inequality is the general form of criterion on the static sideslip stability for conventional airplanes.

\subsection{Using gravity force component as primary control}

The side force component of gravity force caused by the change in roll attitude can be used as primary control. The change in roll attitude $\Delta \Phi$ is obtained by using the aileron, and the steady straight flight is maintained by use of the rudder. In this situation, another derived form of definition on static sideslip stability is given by ${ }^{11)}$ 


$$
\mathrm{d} \Phi / \mathrm{d} \beta>0
$$

This form of definition is the same as the requirement in MIL-F-8785B 3.3.6.2, "Side forces in steady sideslips."7) In this section, it is shown that the definition Eq. (17) is equivalent to the fundamental definition on static sideslip stability Eq. (1).

The equilibrium equations in "FC.0" are given by Eq. (3a) (3c). The equations in "FC.1" are given by

$$
\begin{aligned}
C_{Y 1} & =C_{Y}+C_{Y \beta} \Delta \beta+C_{Y \delta_{a}} \Delta \delta_{a}+C_{Y \delta_{r}} \Delta \delta_{r} \\
& =C_{Y \beta} \Delta \beta+C_{Y \delta_{a}} \Delta \delta_{a}+C_{Y \delta_{r}} \Delta \delta_{r} \\
& =-C_{L 1_{g}} \cos \gamma_{0} \sin \left(\Phi_{0}+\Delta \Phi\right) \\
C_{\ell 1} & =C_{\ell}+C_{\ell \beta} \Delta \beta+C_{\ell \delta_{a}} \Delta \delta_{a}+C_{\ell \delta_{r}} \Delta \delta_{r} \\
& =C_{\ell \beta} \Delta \beta+C_{\ell \delta_{a}} \Delta \delta_{a}+C_{\ell \delta_{r}} \Delta \delta_{r}=0 \\
C_{n 1} & =C_{n}+C_{n \beta} \Delta \beta+C_{n \delta_{a}} \Delta \delta_{a}+C_{n \delta_{r}} \Delta \delta_{r} \\
& =C_{\ell \beta} \Delta \beta+C_{\ell \delta_{a}} \Delta \delta_{a}+C_{\ell \delta_{r}} \Delta \delta_{r}=0
\end{aligned}
$$

By subtracting Eq. (3a) from Eq. (18a), we obtain the increment in the side force component of gravity force from FC.0 to FC. 1 as follows:

$\Delta$ (side force component of gravity force)

$$
\begin{aligned}
& =W \cos \gamma_{0} \sin \left(\Phi_{0}+\Delta \Phi\right)-W \cos \gamma_{0} \sin \Phi_{0} \\
& \cong W \cos \gamma_{0} \cos \Phi_{0} \cdot \Delta \Phi \\
& =\rho U_{0}^{2} S\left(C_{Y \beta} \Delta \beta+C_{Y \delta_{a}} \Delta \delta_{a}+C_{Y \delta_{r}} \Delta \delta_{r}\right) / 2
\end{aligned}
$$

In a comparison of Eq. (19) with Eq. (6), it is obvious that this gravity force component is equal to the nonequilibrium side force $\Delta Y$ in FC.2. Since $W \cos \gamma_{0} \sin \Phi_{0}=$ const. $>$ 0, the definition Eq. (1) can be rewritten to Eq. (17). Thus Eq. (1) is equivalent to Eq. (17). Consequently, the criteria obtained in the previous section can also be applied.

\section{Static Roll Stability}

In this chapter, the details of "static roll stability" are discussed and its stability criteria in terms of the aerodynamic characteristics of airframe are deduced.

We presume FC.0 to be a trimmed, straight, and winglevel flight condition. To give a small change to the roll attitude in FC.0, a rolling moment as an external force is applied to the airplane by using its aileron as primary control; we use its rudder as secondary control to maintain a steady straight flight. As a result, the new steady flight condition FC. 1 is established in which the roll attitude $\Phi_{1}$ is " $\Phi_{0}+\Delta \Phi$." It is then assumed that the flight speed and flight path angle in FC. 1 are equal to those in FC.0, (i.e., $U_{1}=U_{0}$ and $\gamma_{1}=\gamma_{0}$ ).

At FC.2, when the additive aileron deflection $\Delta \delta_{a}$ is quickly returned to zero, if the resulting nonequilibrium rolling moment $\Delta R$ acts to restore the increment in roll attitude $\Delta \Phi$, the static roll stability is stable, and vice versa. Therefore the condition for static roll stability to be stable may be written

$$
\mathrm{d} R / \mathrm{d} \Phi<0
$$

This is the fundamental definition on "static roll stability."

The rolling moment applied by the aileron in FC. 1 balances against $\Delta R$ (i.e., $\left[\rho U_{1}^{2} / 2\right] S b \cdot C_{\ell \delta_{a}} \Delta \delta_{a}=-\Delta R$ ). Consequently, since $C_{\ell \delta_{a}}<0$, the derived form of definition of static roll stability is given by

$$
\mathrm{d} \delta_{a} / \mathrm{d} \Phi<0
$$

This shows that the static stability can be judged by checking the sense of the primary control in FC.1, and that it can be utilized for the quantitative measurements in flight tests.

\subsection{Equilibrium equation and restoring force}

The equilibrium equations in "FC.0" are the same with Eq. (3a) (3c), and the equations in "FC.1" are given by "In FC.1":

Side force: $\quad Y_{1}=-W \cos \gamma_{0} \sin \left(\Phi_{0}+\Delta \Phi\right)$,

$$
\text { or } \begin{aligned}
C_{Y 1} & =C_{Y}+C_{Y \beta} \Delta \beta+C_{Y \delta_{a}} \Delta \delta_{a}+C_{Y \delta_{r}} \Delta \delta_{r} \\
& =-C_{L 1_{g}} \cos \gamma_{0} \sin \left(\Phi_{0}+\Delta \Phi\right) \\
& \cong-C_{L 1_{g}} \cos \gamma_{0}\left(\sin \Phi_{0}+\cos \Phi_{0} \cdot \Delta \Phi\right),
\end{aligned}
$$

or $C_{Y \beta} \Delta \beta+C_{Y \delta_{a}} \Delta \delta_{a}+C_{Y \delta_{r}} \Delta \delta_{r}$

$$
=-C_{L 1_{g}} \cos \gamma_{0} \cos \Phi_{0} \cdot \Delta \Phi
$$

Rolling moment: $\quad R_{1}=0$,

$$
\begin{aligned}
& \text { or } C_{\ell 1}=C_{\ell}+C_{\ell \beta} \Delta \beta+C_{\ell \delta_{a}} \Delta \delta_{a}+C_{\ell \delta_{r}} \Delta \delta_{r}=0, \\
& \text { or } C_{\ell \beta} \Delta \beta+C_{\ell \delta_{a}} \Delta \delta_{a}+C_{\ell \delta_{r}} \Delta \delta_{r}=0
\end{aligned}
$$

Yawing moment: $\quad N_{1}=0$,

$$
\begin{aligned}
& \text { or } C_{n 1}=C_{n}+C_{n \beta} \Delta \beta+C_{n \delta_{a}} \Delta \delta_{a}+C_{n \delta_{r}} \Delta \delta_{r}=0, \\
& \text { or } C_{n \beta} \Delta \beta+C_{n \delta_{a}} \Delta \delta_{a}+C_{n \delta_{r}} \Delta \delta_{r}=0
\end{aligned}
$$

where $C_{L 1_{q}}$ is shown by Eq. (5b).

The nonequilibrium rolling moment $\Delta R$ at FC.2, when the additive aileron deflection $\Delta \delta_{a}$ in FC. 1 is returned to zero, can be obtained by subtracting the aileron effectiveness $C_{\ell \delta_{a}} \Delta \delta_{a}$ from the equilibrium condition " $C_{\ell 1}=0$ " in FC. 1 and is shown by

$$
\begin{aligned}
\Delta R & =\rho U_{0}{ }^{2} S b\left(C_{\ell 1}-C_{\ell \delta_{a}} \Delta \delta_{a}\right) / 2 \\
& =\rho U_{0}{ }^{2} S b\left(C_{\ell \beta} \Delta \beta+C_{\ell \delta_{r}} \Delta \delta_{r}\right) / 2
\end{aligned}
$$

By obtaining the increment in rolling moment $\Delta R_{\delta_{a}}$ ( $\left.=\left[\rho U_{1}^{2} / 2\right] S b C_{\ell \delta_{a}} \Delta \delta_{a}\right)$ produced by the aileron deflection from Eq. (22b), we can reasonably show that this $\Delta R_{\delta_{a}}$ balances against $\Delta R$ (i.e., $\Delta R_{\delta_{a}}=-\Delta R$ ). Therefore, because $C_{\ell \delta_{a}}<0$, the definitions Eq. (20) and Eq. (21) are equivalent.

\subsection{Criteria for static roll stability}

Equation (23) in the previous section shows that $\Delta R$ is a function of $\Delta \beta$ and $\Delta \delta_{r}$. Therefore the definition Eq. (20) can be described by

$$
\frac{\mathrm{d} R}{\mathrm{~d} \Phi}=\frac{\partial R}{\partial \beta} \cdot \frac{\mathrm{d} \beta}{\mathrm{d} \Phi}+\frac{\partial R}{\partial \delta_{r}} \cdot \frac{\mathrm{d} \delta_{r}}{\mathrm{~d} \Phi}<0
$$


The partial differentials in this equation are obtained by differentiating Eq. (23) as follows:

$$
\begin{aligned}
& \partial R / \partial \beta=\rho U_{0}^{2} S b \cdot C_{\ell \beta} / 2 \\
& \partial R / \partial \delta_{r}=\rho U_{0}^{2} S b \cdot C_{\ell \delta_{r}} / 2
\end{aligned}
$$

The total differentials $\mathrm{d} \beta / \mathrm{d} \Phi$ and $\mathrm{d} \delta_{r} / \mathrm{d} \Phi$ in Eq. (24) are deduced by solving the simultaneous equations of Eq. (22a) $\sim(22 \mathrm{c})$ to obtain the ratios of $\Delta \beta$ and $\Delta \delta_{r}$ to $\Delta \Phi$, as follows.

$$
\begin{aligned}
& \mathrm{d} \beta / \mathrm{d} \Phi=\Delta \beta / \Delta \Phi=C_{L 1_{g}}\left(C_{n \delta_{a}} C_{\ell \delta_{r}}-C_{\ell \delta_{a}} C_{n \delta_{r}}\right) / A \\
& \mathrm{~d} \delta_{r} / \mathrm{d} \Phi=\Delta \delta_{r} / \Delta \Phi=C_{L 1_{g}}\left(C_{n \beta} C_{\ell \delta_{a}}-C_{\ell \beta} C_{n \delta_{a}}\right) / A
\end{aligned}
$$

where $A$ is given by Eq. (13).

By applying Eqs. (25a), (25b), (26a), and (26b) for definition Eq. (24), the stability criterion in terms of the aerodynamic characteristics of an airplane is obtained as follows.

$$
\begin{aligned}
& C_{\ell \delta_{a}}\left(C_{n \beta} C_{\ell \delta_{r}}-C_{\ell \beta} C_{n \delta_{r}}\right) /\left\{C_{Y \beta}\left(C_{\ell \delta_{a}} C_{n \delta_{r}}-C_{n \delta_{a}} C_{\ell \delta_{r}}\right)\right. \\
& \quad+C_{\ell \beta}\left(C_{n \delta_{a}} C_{Y \delta_{r}}-C_{Y \delta_{a}} C_{n \delta_{r}}\right) \\
& \left.\quad+C_{n \beta}\left(C_{Y \delta_{a}} C_{\ell \delta_{r}}-C_{\ell \delta_{a}} C_{Y \delta_{r}}\right)\right\}<0
\end{aligned}
$$

When $C_{Y \delta_{a}}, C_{n \delta_{a}}, C_{Y \delta_{r}}$ and $C_{\ell \delta_{r}}$ are small and may be ignored as they are in regard to conventional airplanes, Eq. (27) is simplified as follows.

$$
C_{\ell \beta} / C_{Y \beta}>0
$$

This inequality is the criterion for the static roll stability for conventional airplanes and shows that static roll stability depends not only on "dihedral effect $C_{\ell \beta}<0$," but also on "static sideslip stability $C_{Y \beta}<0$ " mentioned in chapter 2 . This means that if $C_{Y \beta}>0$, the left/right sideslip angle follows the right/left change in roll attitude in FC.1, and $C_{\ell \beta}$ must be positive to generate the restoring rolling moment for the change in roll attitude.

\section{Static Yaw Stability}

In this chapter, the details of "static yaw stability" are discussed and its stability criteria in terms of the aerodynamic characteristics of airframe are deduced.

We presume FC.0 to be a trimmed, straight, and winglevel flight condition. To give a small change to the yaw attitude in FC.0, a yawing moment as an external force is applied to the airplane with its rudder as primary control. We use its aileron as secondary control to maintain a steady straight flight. As a result, the new steady flight condition FC. 1 is established in which the yaw attitude $\Psi_{1}$ is " $\Psi_{0}+$ $\Delta \Psi$." Consequently, this increment in yaw attitude $\Delta \Psi$ is equal to the negative change in sideslip angle in FC.1, as follows:

$$
\Delta \Psi=-\Delta \beta
$$

It is then assumed that the flight speed and flight path angle in FC. 1 are equal to those in FC.0, (i.e., $U_{1}=U_{0}$ and $\gamma_{1}=$ $\left.\gamma_{0}\right)$.

At this FC. 2 when the additive rudder deflection $\Delta \delta_{r}$ is quickly returned to zero, if the resulting nonequilibrium yawing moment $\Delta N$ acts to restore the increment in yaw attitude $\Delta \Psi$, the static yaw stability is stable, and vice versa. Therefore the condition for static yaw stability to be stable is given by

$$
\mathrm{d} N / \mathrm{d} \Psi<0
$$

This is the fundamental definition on "static yaw stability" and can be rewritten by using Eq. (29) as follows:

$$
\mathrm{d} N / \mathrm{d} \beta>0
$$

The yawing moment applied by the rudder in FC. 1 balances against $\Delta N$ (i.e., $\left[\rho U_{1}^{2} / 2\right] S b \cdot C_{n \delta_{r}} \Delta \delta_{r}=-\Delta N$ ). Consequently, since $C_{n \delta_{r}}<0$, the derived form of definition of static yaw stability is given by

$$
\mathrm{d} \delta_{r} / \mathrm{d} \Psi<0
$$

This inequality can be written by applying Eq. (29) as

$$
\mathrm{d} \delta_{r} / \mathrm{d} \beta>0
$$

This form of definition is used for quantitative measurements in flight tests.

\subsection{Equilibrium equation and restoring force}

The equilibrium equations in "FC.0" are equal to Eq. (3a) (3c), and the equations in "FC.1" are given by Eq. (22a) (22c) in section 3.1 .

When the additive rudder deflection $\Delta \delta_{r}$ in FC. 1 is returned to zero, the nonequilibrium yawing moment $\Delta N$ at FC. 2 can be obtained by subtracting the rudder effectiveness $C_{n \delta_{r}} \Delta \delta_{r}$ from the equilibrium condition " $C_{n 1}=0$ " in FC. 1 and is shown by

$$
\begin{aligned}
\Delta N & =\rho U_{0}^{2} \operatorname{Sb}\left(C_{n 1}-C_{n \delta_{r}} \Delta \delta_{r}\right) / 2 \\
& =\rho U_{0}^{2} \operatorname{Sb}\left(C_{n \beta} \Delta \beta+C_{n \delta_{a}} \Delta \delta_{a}\right) / 2
\end{aligned}
$$

By obtaining the increment in yawing moment $\Delta N_{\delta_{r}}$ ( $\left.=\rho U_{0}^{2} S b C_{n \delta_{r}} \Delta \delta_{r} / 2\right)$ produced by the rudder deflection from Eq. (22c), we can reasonably show that this $\Delta N_{\delta_{r}}$ balances against $\Delta N$ (i.e., $\Delta N_{\delta_{r}}=-\Delta N$ ). Since $C_{n \delta_{r}}<0$, the definitions Eq. (30a) and (30b) are respectively equivalent to Eq. (31a) and (31b).

\subsection{Criteria for static roll stability}

Equation (32) in the previous section shows that $\Delta N$ is a function of $\Delta \beta$ and $\Delta \delta_{a}$. Therefore the definition Eq. (30a) may be written

$$
\frac{\mathrm{d} N}{\mathrm{~d} \Psi}=\frac{\partial N}{\partial \beta} \cdot \frac{\mathrm{d} \beta}{\mathrm{d} \Psi}+\frac{\partial N}{\partial \delta_{a}} \cdot \frac{\mathrm{d} \delta_{a}}{\mathrm{~d} \Psi}<0
$$

The partial differentials in this equation are obtained by differentiating Eq. (32) as follows:

$$
\begin{gathered}
\partial N / \partial \beta=\rho U_{0}^{2} S b \cdot C_{n \beta} / 2 \\
\partial N / \partial \delta_{a}=\rho U_{0}^{2} S b \cdot C_{n \delta_{a}} / 2
\end{gathered}
$$


Table 2. Summary of static stability of airplane.

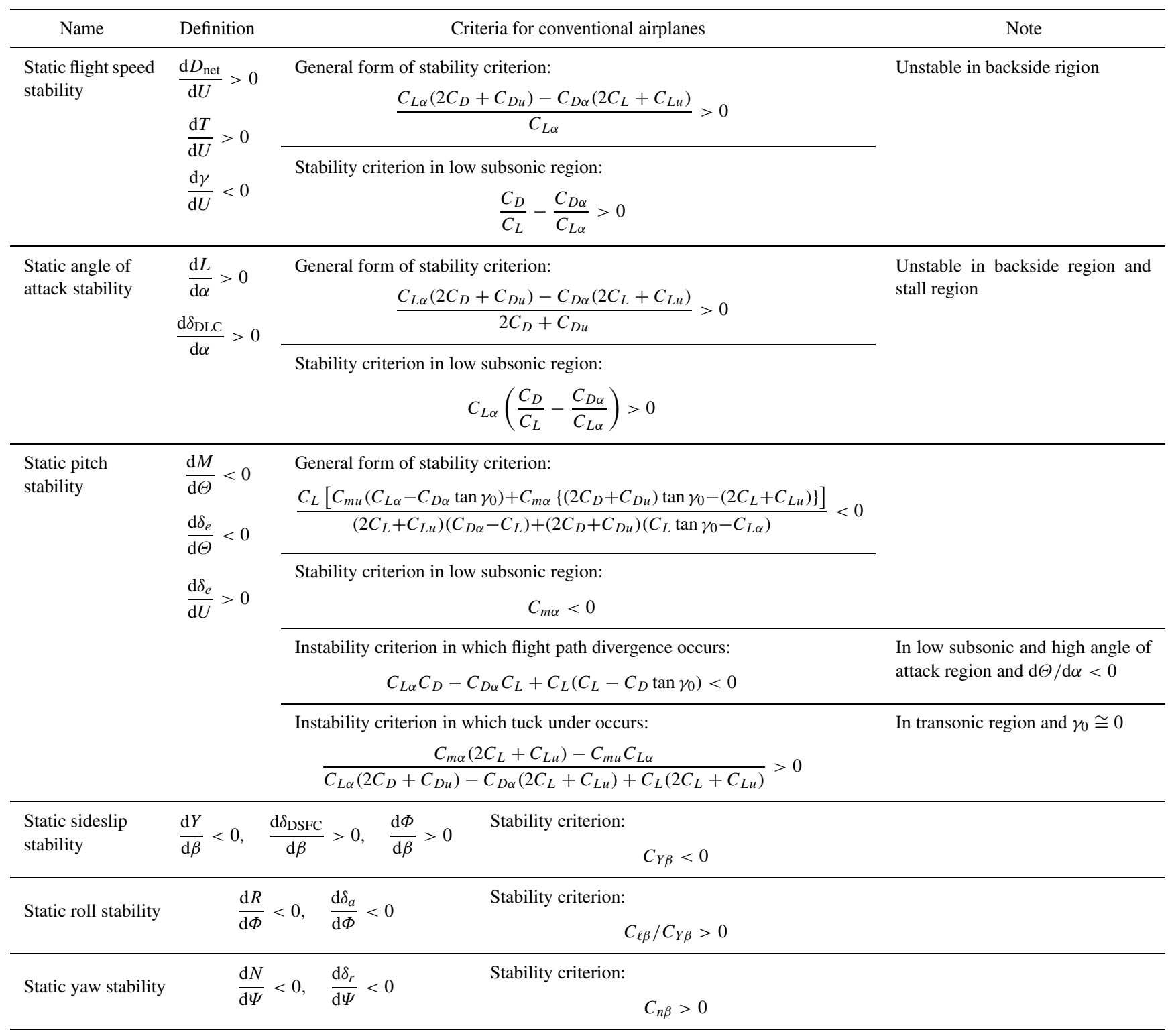

The total differentials $\mathrm{d} \beta / \mathrm{d} \Psi$ and $\mathrm{d} \delta_{a} / \mathrm{d} \Psi$ in Eq. (33) are deduced in this way. At first, the ratios of $\Delta \beta$ and $\Delta \delta_{a}$ to $\Delta \Phi$ are obtained by solving the simultaneous equations of Eq. (22a) $\sim(22 \mathrm{c})$. (Note: $\mathrm{d} \beta / \mathrm{d} \Phi$ is already obtained in Eq. (26a)).

$$
\begin{aligned}
& \mathrm{d} \beta / \mathrm{d} \Phi=\Delta \beta / \Delta \Phi=C_{L 1_{g}}\left(C_{n \delta_{a}} C_{\ell \delta_{r}}-C_{\ell \delta_{a}} C_{n \delta_{r}}\right) / A \\
& \mathrm{~d} \delta_{a} / \mathrm{d} \Phi=\Delta \delta_{a} / \Delta \Phi=C_{L 1_{g}}\left(C_{\ell \beta} C_{n \delta_{r}}-C_{n \beta} C_{\ell \delta_{r}}\right) / A
\end{aligned}
$$

where $A$ is given by Eq. (13).

Thus,

$$
\begin{aligned}
& \frac{\mathrm{d} \beta}{\mathrm{d} \Psi}=\frac{\Delta \beta}{\Delta \Psi}=-1 \\
& \frac{\mathrm{d} \delta_{a}}{\mathrm{~d} \Psi}=-\frac{\mathrm{d} \delta_{a}}{\mathrm{~d} \beta}=-\left(\frac{\Delta \delta_{a}}{\Delta \Phi}\right) /\left(\frac{\Delta \beta}{\Delta \Phi}\right)
\end{aligned}
$$

$$
=\frac{C_{n \beta} C_{\ell \delta_{r}}-C_{\ell \beta} C_{n \delta_{r}}}{C_{n \delta_{a}} C_{\ell \delta_{r}}-C_{\ell \delta_{a}} C_{n \delta_{r}}}
$$

By applying Eqs. (34a), (34b), (36a), and (36b) for definition Eq.(33), we can obtain the following stability criterion in terms of the aerodynamic characteristics of an airplane.

$$
\frac{C_{n \delta_{r}}\left(C_{n \beta} C_{\ell \delta_{a}}-C_{\ell \beta} C_{n \delta_{a}}\right)}{C_{n \delta_{a}} C_{\ell \delta_{r}}-C_{\ell \delta_{a}} C_{n \delta_{r}}}<0
$$

Because the derivatives $C_{Y \delta_{a}}, C_{n \delta_{a}}, C_{Y \delta_{r}}$, and $C_{\ell \delta_{r}}$ are small and may be ignored for conventional airplanes, Eq. (37) is simplified as follows:

$$
C_{n \beta}>0
$$

This inequality is the criterion on the static yaw stability for conventional airplanes and is familiar as "weathercock stability." 


\section{Summary}

This report is the final report of this paper and discussed the details of "static sideslip stability," "static roll stability," and "static yaw stability." It obtained the following results.

(1) The fundamental definition for static sideslip stability is " $\mathrm{d} Y / \mathrm{d} \beta<0$," and its derived forms are " $\mathrm{d} \delta_{\mathrm{DSFC}} / \mathrm{d} \beta>$ 0 " and " $\mathrm{d} \Phi / \mathrm{d} \beta>0$." The criterion deduced from these definitions requires " $C_{Y \beta}<0$ " for an airplane.

(2) The fundamental definition for static roll stability is " $\mathrm{d} R / \mathrm{d} \Phi<0$," and its derived form is " $\mathrm{d} \delta_{a} / \mathrm{d} \Phi<0$." The criterion deduced from these definitions is " $C_{\ell \beta} / C_{Y \beta}>0$ " and depends not only on "dihedral effect $C_{\ell \beta}<0$," but also on "static sideslip stability $C_{Y \beta}<0$."

(3) The fundamental definition for static yaw stability is " $\mathrm{d} N / \mathrm{d} \Psi<0$," and its familiar form is " $\mathrm{d} \delta_{r} / \mathrm{d} \beta>0$ " in steady sideslip flights. The criterion deduced from these definitions requires "weathercock stability $C_{n \beta}>0$ " for an airplane.

The entire study is summarized as follows: This paper proposed the fundamental definitions on static stability for the six degrees of freedom motion, as shown in Table 1. On the basis of these fundamental definitions, each static stability concept was discussed in detail, and the criteria in terms of the aerodynamic characteristics of an airplane were obtained. The results are summarized in Table 2, the most important result being that all the static stability concepts of an airplane can be discussed systematically on the basis of the fundamental definitions mentioned above.

\section{References}

1) Perkins, C. D. and Hage, R. E.: Airplane Performance Stability and Control, John Wiley \& Sons, New York, 1949, p. 11.

2) Etkin, B.: Dynamics of Flight-Stability and Control, John Wiley \& Sons, New York, 1959, pp. 15-16, 27-28.

3) McRuer, D., Ashkenas, I. and Graham, D.: Aircraft Dynamics and Automatic Control, Princeton University Press, Princeton, 1973, pp. 271-273.

4) Anderson, J. D., Jr.: Introduction to Flight, third ed., McGraw-Hill, New York, 1989, pp. 369-374.

5) Japan Society for Aeronautical and Space Sciences: Handbook for Aeronautical and Astronautical Engineering, second ed., Maruzen, Tokyo, 1992, pp. 410-412 (in Japanese).

6) McCormick, B. W.: Aerodynamics Aeronautics and Flight Mechanics, second ed., John Wiley \& Sons, New York, 1995, pp. 474-476.

7) Military Specification : Flying Qualities of Piloted Airplanes, MIL-F8785B(ASG), 1969.

8) Kobayashi, O.: Static and Dynamic Flight-Path Stability of Airplanes, Trans. Jpn. Soc. Aeronaut. Space Sci., 36 (1993), pp. 107-120.

9) Hira, N.: Theory of High Speed Flight, Hirokawa, Tokyo, 1977, p. 132 (in Japanese).

10) Heffley, R. K., Stapleford, R. L. and Rumold, R. C.: Airworthiness Criteria Development for Powered-Lift Aircraft, NASA CR-2791, 1977, pp. 32-33.

11) Kobayashi, O.: Definition System and Criteria Related to the Static Stability of Airplanes (First Report) Definition System, Static Flight Speed Stability and Static Angle of Attack Stability, Trans. Jpn. Soc. Aeronaut. Space Sci., 45 (2002), pp. 116-123.

12) Kobayashi, O.: Definition System and Criteria Related to the Static Stability of Airplane (Second Report) Static Pitch Stability, Trans. Jpn. Soc. Aeronaut. Space Sci., 45 (2002), pp. 124-131. 